\title{
Influencia de la actividad física y práctica deportiva en el rendimiento académico del alumnado de educación secundaria
}

\section{Influence of physical activity and sports practice in the academic performance of secondary education students}

\author{
Saúl Villalba Lombarte', Manuel Villena Serrano ${ }^{2 *}$ y Rosario Castro López ${ }^{3}$ \\ 1 Graduado en Ciencias de la Actividad Física y Deporte. \\ 2 Doctor en Innovación Didáctica y Formación de Profesorado, Universidad de Jaén (España). \\ 3 Universidad Internacional de la Rioja (España).
}

\begin{abstract}
Resumen: La presente investigación pretende identificar si la realización de práctica deportiva y de actividad física tiene influencia en el rendimiento académico de adolescentes en su etapa escolar. Se ha tomado a través de un enfoque cuantitativo con carácter inferencial, una muestra de 91 alumnos estudiantes de ESO ( $n=91)$. Los instrumentos utilizados han sido el cuestionario mundial de actividad física GPAQ (OMS, 2004), valoración subjetiva de la práctica deportiva y una serie de cuestiones sobre el rendimiento académico, centradas en la nota media del último trimestre y número de asignaturas suspendidas. Los resultados muestran que los alumnos que exponen mayores niveles de actividad física tienen un mejor rendimiento académico que los que no realizan dicha práctica. A su vez, estos resultados son más positivos cuantas más horas de práctica deportiva se realizan, y los alumnos que realizan cualquier tipo de práctica deportiva tienen una valoración más positiva que los alumnos inactivos.

Palabras clave: Actividad física, educación física, rendimiento académico, educación, hábitos saludables.
\end{abstract}

Abstract: This research aims to identify whether the performance of sport and physical activity influences the academic achievement of adolescents in their school years. To do this, it has been taken through a quantitative approach to inferential character, a sample of 91 students of ESO students ( $\mathrm{n}$ =91). On the one hand, sport and physical activity was measured through global physical activity questionnaire GPAQ (OMS, 2004), on the other hand, have implemented a number of questions on academic performance, focusing on the overall rating the last quarter and the number of failed subjects in addition to their subjective assessment of sports. The results show that students exhibiting higher levels of physical activity have better academic performance than those not involved in the practice, both in its upper half note as the lower number of failed subjects. In turn, these results are more positive when many hours of sport are made, and students who perform also any kind of sports have a more positive assessment than physically inactive students.

Keywords: Physical activity, sport, physical education, academic performance, education, health behavior, physical exercise.

\section{Introducción}

El deporte y el ejercicio físico ha sido un motor de gran importancia para impulsar la práctica de vida saludable en una sociedad corrompida por una vida de hábitos insanos. Actualmente vivimos en una sociedad basada en el sedentarismo, estrés social y hábitos inadecuados, pero esto no solo afecta a la población adulta, los niños y adolescentes practican muy poca actividad física y estos niveles están disminuyendo en la actualidad (Mollá, 2007). Esto puede deberse a los cambios sociales que se están viviendo basados en la intelectualización provocando así un aumento y desarrollo de la tenologización (Aránguiz, 2009), pues acorde con Martínez, Moreno, Marqués-Lopes y Martí (2002) las actividades que se realizan actualmente conllevan un gasto energético mucho más inferior que en épocas pasadas.

A lo largo de la historia la asignatura de Educación Física ha estado presente en la educación de los más jóvenes. Actual-

Dirección para correspondencia [Correspondence address]: Manuel Villena Serrano. Dirección: C/Andres Segovia no 4, $2^{\circ} \mathrm{B}$ Armilla, Granada (Espańa). E-mail: m_villena_serrano@yahoo.es mente ésta es una asignatura que entre todas sus ventajas y beneficios busca también una educación en valores intentando concienciar al alumno de los beneficios de la práctica deportiva (Perczyk, 2003), además se considera una asignatura motivante debido a su carácter práctico, provocando satisfacción y vivencia autónoma a pesar de sus pocas horas lectivas en comparación con otras asignaturas troncales (Moreno, Cervelló, Zomeño y Marín, 2009). Los centros escolares deben promocionar hábitos positivos para la salud, y animar a los más jóvenes a la realización de actividad física para favorecer una vida más activa (Ortega, Ruiz, Castillo y Sjöström, 2008), además de informar a las familias, pues tienen un papel muy importante en la educación de los nińos y adolescentes para incorporar hábitos beneficiosos para la salud y para la promoción de cualquier actividad (Calzada, 2004).

Acorde con Ortega et al. (2008), en la sociedad de hoy en día tiene especial importancia la práctica deportiva o la realización de cualquier tipo de actividad física extraescolar en edades escolares, a pesar de que no está muy valorada y numerosas investigaciones confirman niveles bajos de actividad 
física y deportiva extraescolar en la actualidad (González y Portolés, 2014). Estas actividades ayudan a combatir problemas cardiovasculares como la hipertensión arterial, la cardiopatía isquémica y los accidentes cerebrovasculares, también ayuda a combatir la obesidad, cuyos niveles en la actualidad están llegando a ser considerados como epidemia (Varo, Martínez y Martínez, 2003).

Otros investigadores como Casajús y Vicente-Rodríguez (2011) muestran que, en los niños y adolescentes, la actividad física tiene beneficios en la adiposidad de los sujetos, por lo tanto, la actividad física se toma como un factor protector de hábitos poco saludables para la salud (Nistal y Prieto, 2003). Además, tal y como afirman Fraile et al. (2004), Mandado y Díaz (2004) y Ruiz y Cabrera (2004) la práctica de actividad física y deportiva sirve como instrumento socializador realizando actividades de forma colectiva que supone en el adolescente una formación integral de todo su ser ayudando también a trabajar una educación en valores relacionados con la solidaridad, cooperación, igualdad de oportunidades, respeto, trato digno etc. Pero la realización de actividad física extraescolar no solo tiene beneficios para la salud física de los adolescentes, sino que también afecta a los procesos cognitivos y de enseñanza-aprendizaje del alumno, pues la velocidad de procesamiento cognitivo es mayor en adolescentes en forma (Hillman, Castelli, Buck, 2005) teniendo también una gran influencia en las funciones psicológicas y sociales (Gutiérrez, 2004). Otros investigadores como Crone, Smith y Gough (2006) han realizado una revisión teórica de la importancia que la actividad física tiene para la población hablando de beneficios para los procesos cognitivos, ansiedad y autoconcepto, actividad cerebral y cambios funcionales en el cerebro y procesos de socialización. El estudio de Villena, Casto, Moreno y Cachón (2015) afirma que la práctica de actividad física en la adolescencia tiene una influencia positiva en el rendimiento académico de los alumnos además Kovacs et al. (2008) añade que fomenta la disciplina y un estilo de vida más saludable, y esta influencia depende del grado de dedicación a la actividad física realizada, es decir, una mayor intensidad se asocia a un mejor rendimiento del alumnado. Moriana, Alós, Alcalá, Pino, Herruzo y Ruiz (2006) afirman que hay una gran diferencia en el rendimiento escolar de los alumnos que realizan actividad extra-académica frente a los que no, obteniendo mejores calificaciones aquellos que sí que realizan alguna actividad.

Siguiendo la línea expuesta, el presente trabajo trata de acercarse y conocer la influencia que tiene la práctica de actividades deportivas en edad escolar como son los deportes extraescolares y cualquier actividad que conlleve la realización de ejercicio físico en el rendimiento académico de alumnos adolescentes. A través de este objetivo general cumpliremos también una serie de objetivos específicos más precisos como: Conocer el tiempo que realizan los alumnos actividad física extraescolar; seguido de, analizar la influencia de la actividad física en edad escolar; y por último valorar el número de asignaturas suspendidas en función de la práctica deportiva extraescolar

\section{Metodología}

Se trata de una investigación de enfoque cuantitativo con un carácter inferencial ya que a partir de la obtención de datos se van a inferir una serie de conclusiones.

\section{Muestra}

La muestra utilizada para la realización de este trabajo fue de 91 alumnos ( $\mathrm{n}=91)$ estudiantes de Educación Secundaria Obligatoria (ESO), concretamente alumnos entre $1^{\circ} \mathrm{ESO}$ y $4^{\circ}$ ESO, 43 alumnas (47.253\%) frente a 48 alumnos (52.747\%), cuyas edades estaban comprendidas entre los 12 años y 16 años, y la media de edad fue 13.989 (D.T. \pm 1.378$)$.

\section{Instrumentos}

Para la realización de este trabajo el instrumento de medida comienza un cuestionario sociodemográfico donde se preguntaba sobre la edad y el sexo de cada uno de los alumnos, posteriormente se dividieron en dos cuestionarios:

La primera parte estaba basada en el Cuestionario Mundial sobre Actividad Física GPAQ (OMS, 2004). Este cuestionario mundial sobre la actividad física ha sido desarrollado por la OMS para la vigilancia de la actividad física en los países. Estas preguntas fueron:

- ¿En su tiempo libre, practica usted deportes/fitness intensos que implican una aceleración importante de la respiración o del ritmo cardíaco como [correr, jugar al fútbol] durante al menos 10 minutos consecutivos? ( $\mathrm{Si}$ su respuesta es no, pasar a la pregunta $4^{\circ}$ )

- En una semana típica, ¿cuántos días practica usted deportes/fitness intensos en su tiempo libre?

- En uno de esos días en los que practica deportes/fitness intensos, ¿cuánto tiempo suele dedicar a esas actividades?

La segunda parte consistía en preguntas para conocer el rendimiento académico del alumno a nivel global, las dos primeras preguntas tenían un carácter objetivo mientras que la última tenía un carácter subjetivo. Estas preguntas fueron:

- Nota media del último trimestre:

- Número de asignaturas NO aprobadas en el último trimestre:

- ¿Cómo crees que afecta la práctica de actividad física al rendimiento académico? Positivamente/ Negativamente/ No afecta. 


\section{Procedimiento}

El proceso que se realizó para obtener los datos necesarios se llevó a cabo durante 3 días pasando encuestas a los estudiantes que formaban la muestra de la investigación, parte de estos estudiantes son alumnos del colegio zaragozano O.D. Sto. Domingo de Silos mientras que otra parte de los estudiantes provenían de otros colegios de Zaragoza, todos ellos fueron elegido de manera aleatoria sin existir distinciones entre ellos.

\section{Análisis de datos}

Tras la recogida de la información a través de las encuestas, los datos fueron tratados con el programa SPSS Statistics 19. Este análisis se basó en los principios de estadística descriptiva buscando conocer las características de los datos obtenidos y exponerlos mediante tablas y gráficos. Para conocer la representatividad de las comparaciones realizadas se utilizó la significación bilateral de la prueba de independía chi-cuadrado de Pearson para determinar si existe o no relación entre las variables, para ello se tomó como variables significativas cuando $p<.05$ mientras que las variables no significativas $\mathrm{y}$ por lo tanto no representativas cuando $p>.05$.

\section{Resultados}

A la hora de valorar la nota media de los alumnos que realizan práctica deportiva y los que no realizan, en la Figura 1. vemos que los que realizan práctica deportiva tuvieron una nota media del último trimestre superior $(M=6.64)$ a los alumnos que no realizan $(M=5.91)$, pero estos datos no son significativos debido a que $p=.146(p>.05)$ por lo que no son datos significativos.

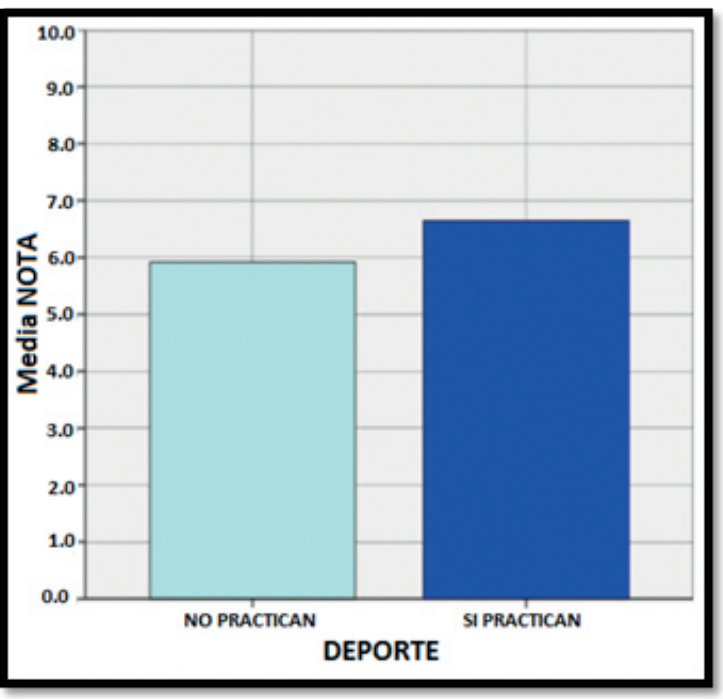

Figura 1. Relación de la nota media del último trimestre entre los alumnos que realizan práctica deportiva con los que no realizan.
En la Figura 2., a pesar de ser no se han encontrado diferencias estadísticamente significativas se puede observar que el número de suspensos fue mayor en alumnos que no realizan práctica deportiva $(M=2.00)$ frente a los alumnos que si realizan práctica deportiva $(M=.96)$, si lo comparamos con la tabla 1 se observa que hay relación entre la comparación de notas suspendidas y nota media del último trimestre.

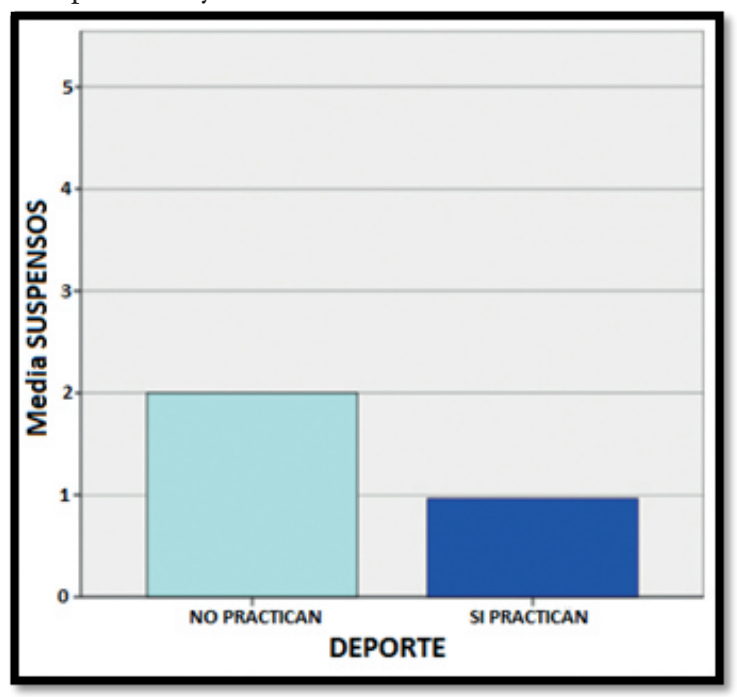

Figura 2. Relación entre la media de suspensos y los alumnos que realizan práctica deportiva con los que no realizan.

Con respecto la Figura 3 se observa que la mayor parte de los alumnos que realizan práctica deportiva tuvieron una valoración positiva de la actividad física $(n=39)$ al igual que los alumnos que no realizan actividad física $(n=15)$. Sin embargo, se encontró un número mayor de alumnos que no realizan práctica deportiva que pusieron que no afecta $(n=21)$ con respecto a los que realizan actividad física $(n=13)$, muy pocos alumnos valoraron negativamente la actividad física. Estos datos se pueden considerar estadísticamente significativos debido a que $p=.007(p<.05)$.

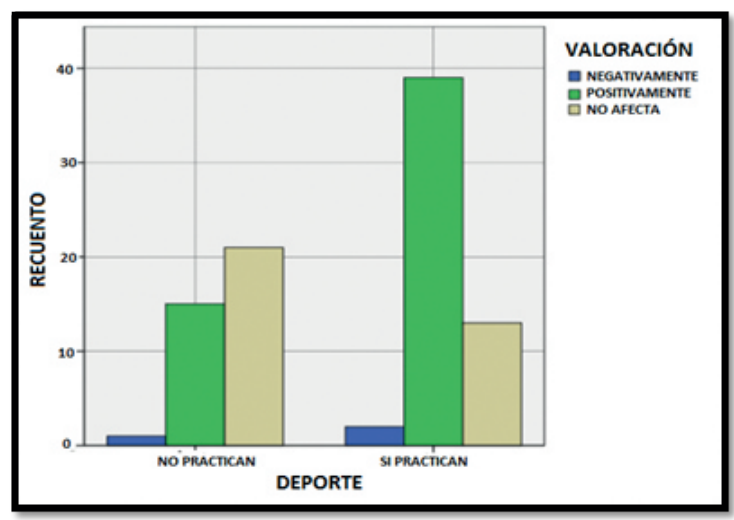

Figura 3. Valoración subjetiva sobre la práctica deportiva de los alumnos que realizan práctica deportiva y los que no realizan. 
Con respecto a la Figura 4 se han dividido a los alumnos que realizan actividad física en tres variables, de 0-5 horas de actividad física a la semana, de 5-10 horas y de 10-15 horas. Se observa que los alumnos que realizan más actividad física (10-15 horas) tuvieron una leve mejora en la nota media del último trimestre $(M=6.95)$ mientras que los que menos actividad física realizan ( $0-5$ horas) tuvieron peor nota media $(M=6.577)$, mientras que los alumnos que realizan 5-10 horas obtuvieron una nota media más cercana a los alumnos que menos horas realizan $(M=6.67)$. Estos datos son significativos debido a que $p=.017(p<.05)$.

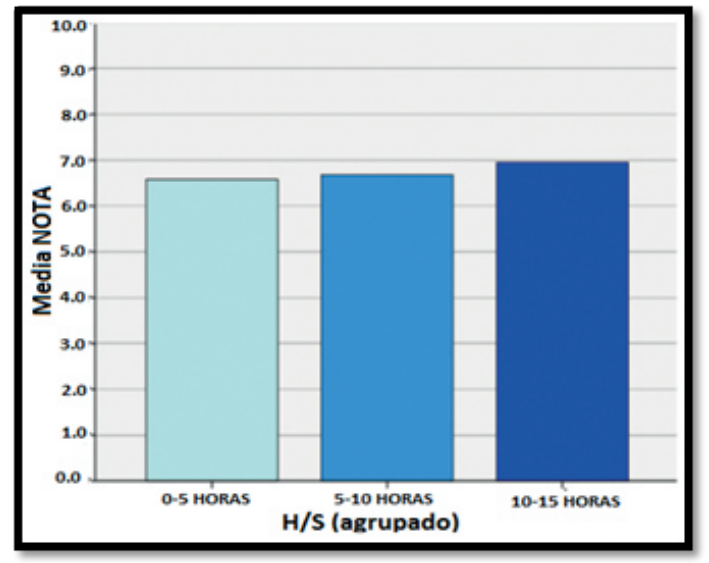

Figura 4. Relación de la nota media entre los alumnos que realizan más actividad física y los alumnos que menos.

En la Figura 5 se observa que los alumnos que realizan más actividad física a la semana (5-10 horas) tuvieron menos asignaturas suspendidas en el último trimestre $(M=.25)$ mientras que los alumnos que realizan $0-5$ horas de actividad física obtuvieron peor resultado $(M=.93)$ y los que realizan 10-15 horas semanales tuvieron resultados muy similares a los anteriores, aunque ligeramente superiores $(M=1.15)$. A pesar de estas diferencias los resultados no resultaron estadísticamente significativos $\mathrm{p}>.05$

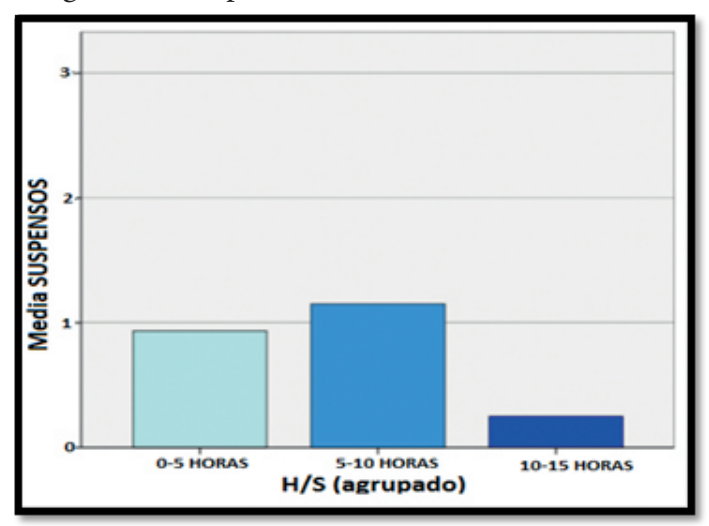

Figura 5. Relación de la media de suspensos entre los alumnos que realizan más actividad física y los alumnos que menos.
En la Figura 6 se puede observar que los alumnos que realizan práctica deportiva tienen mejor nota media en todas las edades $(M 12=7.6 ; M 13=6.4 ; M 14=6.23 ; M 15=6.66)$ con respecto a la nota media de los alumnos que no realizan práctica deportiva $(M 12=6.44 ; M 13=4.79 ; M 14=5.75 ; M 15=6.14)$ excepto con 16 años que obtuvieron una nota media similar entre los alumnos que realizan actividad física y los que no $(M 16=6.86)$. Estos datos no son estadísticamente significativos ( $p>.05)$.

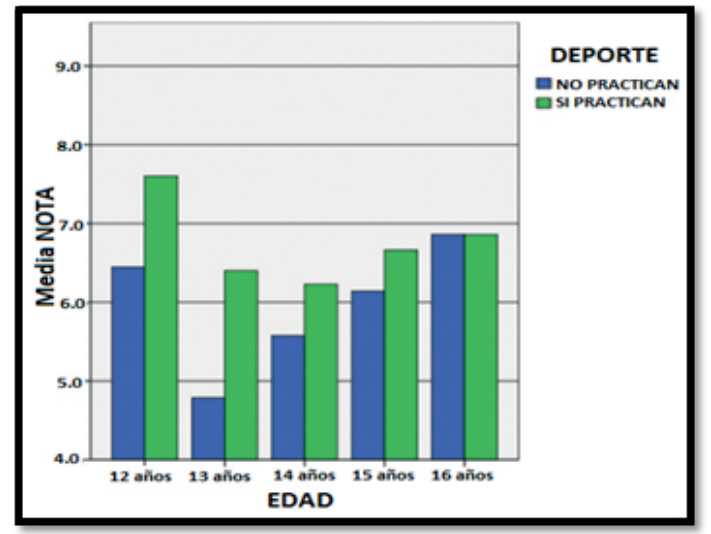

Figura 6. Relación entre la nota media y los alumnos que realizan práctica deportiva con los que no realizan dividido por edades.

En la Figura 7 se puede observar que los alumnos que realizan práctica deportiva tuvieron menos suspensos de media en el último trimestre $(M 12=0 ; M 13=1.69 ; M 14=1.18$; $M 15=.77)$ con respecto a los alumnos que no realizan práctica deportiva $(M 12=1.36 ; M 13=4 ; M 14=2.62 ; M 15=.80)$, a pesar de que los alumnos de 16 ańos que realizan actividad física tuvieron más suspensos de media $(M 16=.58)$ que los alumnos que no realizan actividad física $(M 16=.40)$. Cabe destacar que no son datos estadísticamente significativos ( $p>05)$.

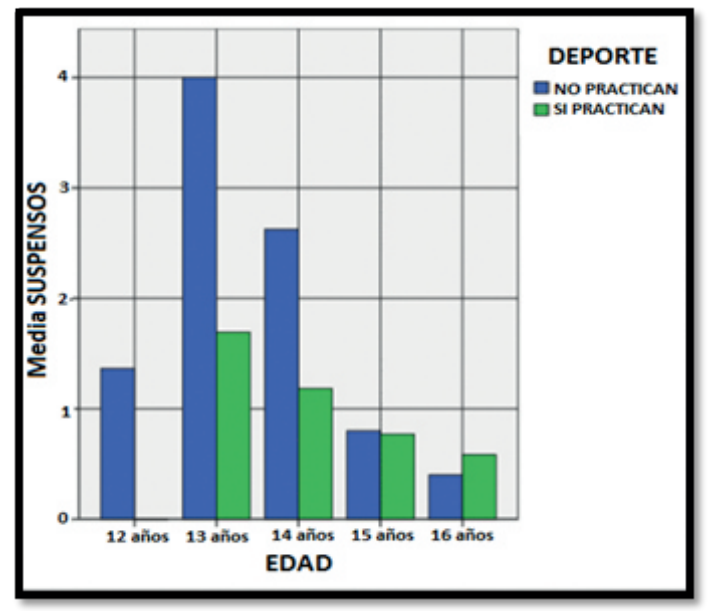

Figura 7. Relación entre la media de asignaturas suspendidas y los alumnos que realizan práctica deportiva con los que no realizan dividido por edades. 
En la Figura 8 se puede observar que en todas las edades se valora positivamente la realización de actividad física, siendo de interés que con 14 años hay un pequeño número de alumnos $(n=3)$ que valora la práctica deportiva de manera negativa. Estos datos también se pueden considerar estadísticamente significativos debido a que $p=.041(p<.05)$.

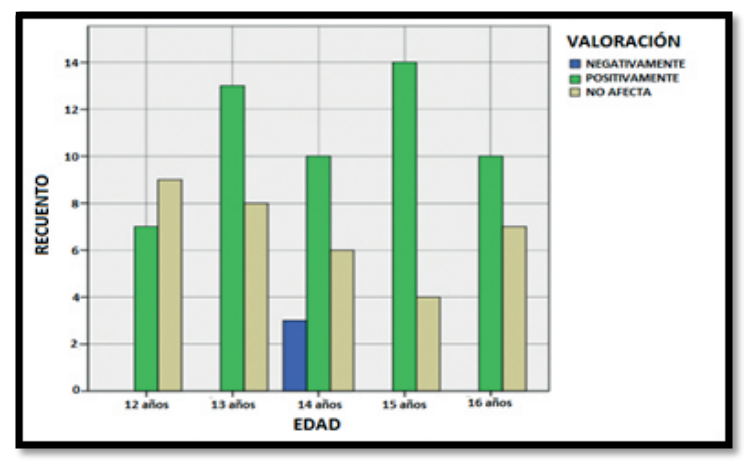

Figura 8. Valoración subjetiva sobre la práctica deportiva de los alumnos divididos por edades.

\section{Discusión}

En primer lugar, al utilizar la variable independiente de si los alumnos realizan o no práctica deportiva, se ha obtenido que los alumnos que practican actividad física han obtenido mejores resultados académicos, teniendo una nota superior en el último trimestre que los alumnos sedentarios, es decir, aquellos alumnos que no realizan ningún tipo de actividad deportiva, al igual que el estudio de Carmona et al. (2011) donde los alumnos que realizan práctica deportiva obtuvieron mejores notas en determinadas asignaturas obligatorias. Estos resultados son similares a los obtenidos en estudios realizados por Ortega et al. (2008), afirmando que los alumnos físicamente activos, además de tener muchos más beneficios para su salud, tienen mejor nivel y rendimiento académico. Hay que tener en cuenta que este dato no se puede considerar estadísticamente significativo ( $p>.05)$ pues posiblemente han afectado otras variables que no han sido tenidas en cuenta a lo largo de este trabajo

Al comparar estos dos grupos en función de las asignaturas suspendidas en el último trimestre se obtienen datos relacionados con los anteriores, los alumnos que realizan práctica deportiva han suspendido menos asignaturas en el último trimestre que los alumnos que no realizan ningún tipo de práctica deportiva, lo que supone un mejor rendimiento académi$c o$, a pesar de que estos datos no sean significativos ( $p>.05)$.

Si tomamos la valoración subjetiva que tiene los alumnos sobre la práctica deportiva, se muestra que los que realizan actividad física tienen una valoración más positiva que los alumnos que no realizan, posiblemente sepan que la realización de estas actividades les favorece personalmente en su rendimiento académico, considerándose estos datos estadísticamente significativos $(p<.05)$.

A su vez, si solo se tienen en cuenta a los alumnos que realizan actividad física, se concluye que cuanto mayor es el número de horas que se realiza práctica deportiva, mayor es la nota media en el último trimestre y por lo tanto mejor es el rendimiento académico obtenido, siendo además estos datos estadísticamente significativos $(p<.05)$. Acorde a estos resultados, el estudio realizado por Kovacs et al. (2008) afirma que existe una relación entre los beneficios en el rendimiento académico y el grado de dedicación de la práctica de actividad física que se realice donde los alumnos que más actividad física realizan obtienen mejores resultados escolares. Además, se puede observar que los alumnos que más horas de práctica deportiva realizan (10-15 horas) tienen un número mucho más inferior de asignaturas suspendidas que la de los compańeros que realizan menos horas de actividad física, a pesar de que estos últimos datos no se pueden considerar estadísticamente significativos ( $p>.05)$.

Si se tiene en cuenta como variable independiente la edad de los alumnos se ha obtenido que en todas las edades, los alumnos que realizan práctica deportiva tienen mejores notas de media en el último trimestre que los alumnos que no realizan ningún tipo de actividad y por lo tanto los alumnos físicamente activos han suspendido menos asignaturas, sin embargo, a los 16 ańos estos resultados tienden a igualarse, puede deberse a una posible disminución de la práctica deportiva por parte de los alumnos o al incremento de la dificultad del nivel educativo en el que se encuentran, pues como afirma Garita(2006) a lo largo de la adolescencia los niveles de actividad física de ambos sexos disminuye considerablemente. Cabe destacar que estos datos sobre la nota media del último trimestre y el número de asignaturas suspendidas no son estadísticamente significativos ( $p>.05)$.

Si se valora la valoración subjetiva de la realización de la actividad física en la influencia en el rendimiento académico se observa que en todas las edades la práctica deportiva está valorada positivamente, pero como resultado llamativo cabe destacar los sujetos de 12 años, pues hay más alumnos que consideran que la realización de práctica deportiva no afecta al rendimiento académico, estos son alumnos de los primeros niveles de Educación Secundario Obligatoria (ESO) por lo que podría deberse a que debido a su corta edad, quizás aún no conozcan todos los beneficios que conlleva la actividad física y la influencia positiva para la salud. Estos datos pueden considerarse estadísticamente significativos $(p<.05)$.

\section{Conclusiones}

Como se ha podido observar a lo largo de la investigación, la práctica deportiva es de vital importancia en todas las etapas de la vida, pero especialmente cobra verdadero interés en la 
edad escolar donde se considera muy importancia la realización de actividad física tanto en niños como en adolescentes para un correcto crecimiento físico y cognitivo.

En el presente trabajo se puede concluir que la práctica deportiva afecta positivamente en la edad escolar, concretamente afecta al rendimiento escolar ya que los alumnos que realizan mayor actividad física han obtenido mejores resultados escolares que los alumnos que no realizan actividad física.

Hay que seguir trabajando y dando a conocer la importan- cia de la actividad física en la actualidad para evitar problemas en la salud de las personas tales como la inactividad física o el sedentarismo. Esto es un objetivo que ningún profesional de la educación y de la actividad física debe olvidar pues tiene que trabajar intentando siempre cumplir con estos objetivos. Desde el presente trabajo se anima a realizar futuras investigaciones para seguir construyendo el futuro de la actividad física para conseguir un mundo más saludable luchando contra los problemas que está teniendo la sociedad.

\section{Referencias}

1. Aránguiz, H. (2009). El sedentarismo no es sólo causa de la tegnologización. Educación física y deporte, 23(1), 33-34.

2. Calzada-Arija, A. (2004). Deporte y educación. Revista de educación, 335, 45-60.

3. Carmona-Rodríguez, C., Sánchez-Delgado, P. y Bakieva, M. (2011). Actividades extraescolares y rendimiento académico: Diferencias en autoconcepto y género. RIE: Revista de Investigación Educativa, 29(2), 447-465.

4. Casajús, J.A. y Vicente-Rodríguez, G. (2011). Ejercicio físico y salud en poblaciones especiales. Exernet. Madrid: Colecciones ICD.

5. Crone, D. Smith, A. y Gough, B. (2006). The physical activity and mental health relationship-a contemporary perspective from qualitative research. Acta universitatis Palackianae Olomucensis, gymnica, 36(3), 29 35.

6. Fraile, A., Álamo, J.M., van den Bergh, K., González, J., Graça, A., Kirk, D., de Knop, P., Lombardozzi, A., Macazaga, A. M., Monjas, R., Romero, S. y Theeboom, M. (2004). El deporte escolar en el siglo XXI: Análisis y debate desde una perspectiva europea (Trabajo de fin de Grado). Universidad de Sevilla, Sevilla.

7. Garita-Azofeifa, E. (2006). Motivos de participación y satisfacción en la actividad física, el ejercicio físico y el deporte. MHSalud: Revista en Ciencias del Movimiento Humano y Salud, 3(1), 1-16.

8. González, J. y Portolés, A. (2014). Actividad física extraescolar: Relaciones con la motivación educativa, rendimiento académico y conductas asociadas a la salud. Revista iberoamericana de psicología del ejercicio y el deporte, 9(1), 51-65.

9. Gutiérrez-Sanmartín, M. (2004). El valor del deporte en la educación integral del ser humano. Revista de educación, 335, 105-126.

10. Hillman, C. H., Castelli, D. M., y Buck, S. M. (2005). Aerobic fitness and neurocognitive function in healthy preadolescent children. Medicine and science in sports and exercise, 37(11), 1-8.

11. Kovacs, F. M., del Real, M. T.G., Gestoso, M., López, J., Mufraggi, N. y Palou, P. (2008). Relación entre hábitos de vida y calificaciones escolares en adolescentes. Apunts. Medicina de l'Esport, 43(160), 181-188.

12. Mandado, A. y Díaz, P. (2004). Deporte y educación: pautas para hacer compatible el rendimiento y el desarrollo integral de los jóvenes depor-

tistas. Revista de Educación, 335, 35-44.

13. Martínez, J.A., Moreno, M.J., Marqués-Lopes, I. y Martí, A. (2002). Causas de la obesidad. Anales del sistema sanitario de Navarra, 25(1), 17-27.

14. Mollá-Serrano, M. (2007). La influencia de las actividades extraescolares en los hábitos deportivos de los escolares. Revista Internacional de Medicina y Ciencias de la Actividad Física y el Deporte, 7(27), 241-252.

15. Moreno-Murcia, J.A., Cervelló-Gimeno, E., Zomeño-Álvarez, T.E. y Marín-de-Oliveria, L.M. (2009). Predicción de las razones de disciplina en educación física. Acción psicológica, 6(2), 7-15.

16. Moriana-Elvira, J.A., Alós-Cívico, F., Alcalá-Cabrera, R., Pino-Osuna, M. J., Herruzo-Cabrera, J. y Ruiz-Olivares, R. (2006). Actividades extraescolares y rendimiento académico en alumnos de Educación Secundaria. Revista electrónica de investigación psicoeducativa, 4(8), 35-46.

17. Nistal-Hernández, P. y Prieto-Saborit, J.A. (2003). La importancia del deporte-salud. Efdeportes, 61.

18. OMS: Departamento de Enfermedades crónicas y Promoción de la Salud (2004). Cuestionario Mundial sobre Actividad Física (GPAQ). Recuperado el 23 de abril de 2014 en http://www.sdprc.org/lhn-tools/ gpaq-spanish.pdf

19. Ortega, F. B., Ruiz, J. R., Castillo, M. J., y Sjöström, M. (2008). Physical fitness in childhood and adolescence: a powerful marker of health. International Journal of Obesity, 32(1), 1-11.

20. Perczyk, J. (2003). El deporte ¡̨es un contenido a enseñar por la escuela?. Efdeportes, 57.

21. Ruiz-Llamas, G. y Cabrera-Suárez, D. (2004). Los valores en el deporte. Revista de educación, 335, 9-19.

22. Varo-Cenarruzabeitia, J.J., Martínez-Hernández, J.A. y MartínezGonzález, M.A. (2003). Beneficios de la actividad física y riesgos del sedentarismo. Medicina Clínica, 121(17), 665-672.

23. Villena-Serrano, M., Castro-López, C. Moreno-Pulido, R., y CachónZagalaz, J. (2015). Estudio comparativo del rendimiento académico y la actividad física en dos institutos de enseńanza secundaria de Andalucía (España). Sport TK: Revista Euroamericana de Ciencias del Deporte, 4(2), $11-18$. 